\section{Is there a link between COQ6 and schwannomatosis?}

To the Editor: Schwannomatosis (OMIM 162091) is a lateonset tumor-predisposition syndrome characterized by the development of multiple nonvestibular, nonintradermal schwannomas. These tumors typically display a molecular signature characterized by biallelic somatic mutations of the NF2 gene in the absence of NF2 germline mutations.

Constitutional heterozygous loss-of-function mutations in SMARCB1 have been reported in less than $10 \%$ of sporadic and in $50 \%$ of familial schwannomatosis cases. The LZTR1 gene was recently found to be mutated in about $80 \%$ of patients with SMARCB1-negative Schwannomatosis. ${ }^{1}$ Nevertheless, the remaining cases still lack a genetic diagnosis, suggesting the existence of other loci predisposing to schwannomatosis.

The SMARCB1/SNF5/INI1 gene encodes a core subunit of the SWI/SNF adenosine triphosphate-dependent chromatin remodeling complex. The product encoded by the LZTR1 gene belongs to a superfamily of BTB/POZ proteins involved in fundamental cellular processes, ranging from the regulation of cytoskeleton dynamics to the control of the cell cycle.

The article "A Germline Missense Mutation in COQ6 Is Associated with Susceptibility to Familial Schwannomatosis," by Zhang et al., described a novel mutation in the coenzyme Q10 (CoQ) biosynthetic gene COQ6 associated with susceptibility to familial schwannomatosis. They performed wholeexome/genome sequencing on genomic DNA from affected and healthy members of a large family with schwannomatosis without constitutional mutations in SMARCB1 and LZTR1. They identified the novel heterozygous missense mutation c.622G >C p.(Asp208His) in the COQ6 gene, segregating with the disease, and demonstrated that it abolishes the ability of the human COQ6 gene to complement a coq6-deficient yeast strain. We believe that these data should be interpreted with caution and that appropriate experimental models are necessary to validate novel disease associations.

The pedigree of the family reported by Zhang et al. ${ }^{2}$ clearly suggests dominant inheritance, which has been reported in most forms of familial schwannomatosis described so far. The probability that the COQ6 variant in this family is segregating just by chance is $1: 128$, and the number of variants identified by exome sequencing is so large that the analysis of a small family may yield several such variants. In fact, the authors identified a total of 12 heterozygous variants, including 9 missense variants, all segregating in the affected members and absent in the healthy individuals. A possible pathogenetic role of the other variants was not excluded.
Importantly, both the genetic and the immunohistochemical analyses of schwannomas from the affected patients did not reveal any alteration in NF2. Moreover, Zhang et al. ${ }^{2}$ did not provide any evidence of possible biologically plausible mechanism(s) linking the haploinsufficiency of COQ6 to oncogenesis in Schwann cells. COQ6 encodes a monooxygenase required for the C5-hydroxylation of the quinone ring of CoQ. We previously characterized the human COQ6 gene, showing that its function is highly conserved, as indicated by its ability to complement the defective respiratory phenotype of a coq6-deleted strain. ${ }^{3}$

The yeast model used by Zhang et al. ${ }^{2}$ simply proved that the p.Asp208His substitution causes a loss of function in the COQ6 gene, as revealed by the lack of complementation in the defective coq6-yeast strain. However, all known mutations in the COQ6 gene are inactivating and have been reported so far in patients affected by an autosomal recessive multisystemic syndrome characterized by nephrotic syndrome and sensorineural deafness. ${ }^{3}$ Conversely, to date there is no evidence that haploinsufficiency of COQ6 causes significant CoQ deficiency; accordingly, none of the patients or their heterozygous parents, even those carrying null alleles, develop schwannomas. Among genes required for CoQ biosynthesis, the only one whose haploinsufficiency has been related to a human disorder is COQ4; however, this gene does not seem to have known enzymatic functions. It seems instead to be involved in the assembly and regulation of the CoQ biosynthetic complex.

Furthermore, knockdown of COQ6 in a cellular model does not reproduce the situation observed in cells of heterozygous patients. In fact, COQ6 is not a limiting factor in CoQ biosynthesis, and Zhang et al. ${ }^{2}$ observed an increase in reactive oxygen species only when knockdown reduced residual messenger RNA (and possibly protein) concentrations to values significantly inferior to $50 \%$ of the wild type, a situation resembling that in cells with homozygous hypomorphic alleles. ${ }^{3}$

A possible dominant-negative effect or a toxic gain of function of the missense COQ6 variant, which would explain the dominant inheritance, was not demonstrated by the experimental model used by Zhang et al. ${ }^{2}$ Moreover, none of the affected members they described showed any symptoms of CoQ deficiency.

Mitochondrial enzymes may act as antioncogenes (complex II subunits or fumarate hydratase are classical examples), but in these cases there is always loss of heterozygosis in the tumor cells. In this case molecular analysis of schwannoma tissues from the affected individuals excluded loss of heterozygosity, and other alterations of the COQ6 gene were not identified. This is in contrast with what it is usually found in schwannomatosis tumors related to SMARCB1 or LZTR1. In fact, both genes act as oncosuppressors; accordingly, tumors from patients with schwannomatosis typically show biallelic loss of the NF2 gene and loss of heterozygosity of SMARCB1 or LZTR1 with retention of the germline mutation. ${ }^{1}$ 
Overall, these data only demonstrate that the p.Asp208His substitution is a "typical" loss-of-function COQ6 mutation; they do not provide any explanation of why it is associated with schwannomatosis, and it is not possible to exclude that this variant is just an incidental finding unrelated to the disease is not possible.

Schwannomatosis requires adequate follow-up to promptly detect novel schwannomas and possible complications. The identification of a novel gene associated with schwannomatosis has important implications in genetic counseling because it allows both the prenatal and the presymptomatic diagnosis of this adult-onset condition in at-risk family members.

Unless further experimental evidence explaining the link between the COQ6 heterozygous missense mutation and the susceptibility to schwannomatosis is provided, we are skeptical about the opportunity of screening for the COQ6 gene in patients affected by schwannomatosis (and screening for schwannomas in individuals with heterozygous mutations in COQ6).

\section{Open}

\section{Response to Trevisson et al.}

To the Editor: First, we thank Trevisson et al. ${ }^{1}$ for their valuable contribution to this subject and their comments on our brief report. In our study, we reported a familiar schwannomatosis without mutations of SMARCB1/INI1/hSNF5 and LZTR, the two known causative genes for schwannomatosis. Using genome and exome sequencing, we found a heterozygous lossof-function mutation of the coenzyme Q10 (CoQ10) biosynthesis monooxygenase 6 (COQ6) gene in patients affected by disease. ${ }^{2}$ CoQ10 is an electron carrier in the mitochondrial respiratory chain, as well as a lipid-soluble antioxidant implicated in protecting cells from damage by reactive oxygen species. Its biosynthesis is still not well characterized in human cells. Mutations in CoQ10 biosynthesis genes, including COQ4 and COQ6, cause primary CoQ10 deficiency. The manifestations of primary CoQ10 deficiency are very heterogeneous, and CoQ10 deficiency has been involved in many common disorders with increased oxidative stress, such as neurodegenerative diseases, cancer, cardiovascular diseases, diabetes mellitus, aging, and Alzheimer disease. It is well known that chronic increases in oxidative stress may trigger transformation and contribute to cancer progression by amplifying genomic instability. We proposed that the halpoinsufficiency of COQ6 monooxygenase due to a loss-of-function mutation may lead to CoQ10 deficiency and chronic overproduction of reactive oxygen species in Schwann cells, thereby predisposing to schwannomatosis.

A critical issue of the hypothesis is whether heterozygous loss-of-function COQ6 causes haploinsufficiency. A previous study of yeast found that haploinsufficiency of COQ6 resulted in a mild reduction of fitness in a medium containing glucose. ${ }^{3}$ Moreover, we assumed that the haploinsufficiency of the COQ6 gene and CoQ10 deficiency may be conditional, dynamic, and tissue/cell specific. For example, one study found that cellular

\section{DISCLOSURE}

The authors declare no conflict of interest.

\author{
Eva Trevisson, $\mathrm{MD}, \mathrm{PhD}^{1}$, Maurizio Clementi, $M D^{1}$ and \\ Leonardo Salviati, $M D, P h D^{1}$
}

${ }^{1}$ Department of Woman and Child Health, Clinical Genetics Unit, University of Padova, Padua, Italy. Correspondence: Eva Trevisson (eva.trevisson@unipd.it)

\section{REFERENCES}

1. Piotrowski A, Xie J, Liu YF, et al. Germline loss-of-function mutations in LZTR1 predispose to an inherited disorder of multiple schwannomas. Nat Genet 2014;46:182-187.

2. Zhang K, Lin JW, Wang J, et al. A germline missense mutation in COQ6 is associated with susceptibility to familial schwannomatosis. Genet Med 2014; 16:787-792.

3. Doimo M, Trevisson E, Airik R, et al. Effect of vanillic acid on COQ6 mutants identified in patients with coenzyme Q10 deficiency. Biochim Biophys Acta 2014;1842:1-6.

doi:10.1038/gim.2014.211

and tissue concentrations of CoQ10 decrease with age, and cellular concentrations below a critical threshold are incompatible with life. Furthermore, carcinogenesis is determined not only by genetic alterations but also gene-environment interaction, as well as metabolism, and not all transgenic mice with the exactly same genetic background and alterations may develop cancers. In addition, germ-line abnormalities associated with cancer may be detected in every cell in the body or only in the tumor cells. Interestingly, despite the presence of a constitutional genetic abnormality that might affect growth regulatory pathways in all cells, people are generally predisposed to only certain tumor types. The average age at disease onset was $\sim 40$ years for this familial schwannomatosis, indicating a chronic disease progression. In this particular family, we considered that the loss-of-function COQ6 allele may lead to a chronic or conditional haploinsufficiency of COQ6 in a cell/tissue-specific manner.

So far, the roles of mutations of CoQ10 biosynthetic genes in cancers are completely unknown. The association is only now coming of age, for example, the most recent cancer genomic studies identify COQ2 gene mutations in human melanoma, colon and rectal cancers, ovarian carcinoma, and glioblastoma multiforme. In addition, more than 48 missense mutations of the COQ6 gene have been identified in various human cancers (http://cancer.sanger.ac.uk/cancergenome/projects/cosmic). Furthermore, abnormally low plasma concentrations of CoQ10 have been found in a number of cancer types, including cervical cancer, breast cancer, and melanoma. ${ }^{4}$ Although intensive research is needed to further explore the underlying mechanisms, increasing data have strongly indicated an undetermined implication of CoQ10 biosynthesis gene mutations in carcinogenesis.

Indeed, the exact oncogenic mechanism of the loss-offunction COQ6 gene in disease remains a challenging question to be elucidated. Cancer is a complex multigenic disease 